\title{
A Method Based on Fuzzy Logic Technique for Smoothing in 2D
}

\author{
Ahmet Çinar \\ F1rat University, Faculty of Engineering, Computer Engineering, 23119 Elazig, \\ Turkey \\ acinar@firat.edu.tr
}

\begin{abstract}
This paper describes a novel approach based on fuzzy logic technique for smoothing process on the unstructured meshes in 2D. The proposed method works local on triangulation. Therefore, remeshing does not require. It is known that other smoothing operations such as laplacian smoothing, optimization based smoothing, angle based smoothing, and hybrid smoothing operations work global. That is, in these methods, meshing is achieved, afterwards remeshing is done for smoothing. Whereas, the goal of presented method is to find the best fit location of node while meshing is done. However, remeshing process is avoided.
\end{abstract}

\section{Introduction}

The basic element in unstructured mesh generation in 2D is triangle and quadrangle. Triangulation is known as creating mesh by using triangles[1]. For optimum triangulation, elements of the created unstructured mesh must be equal size. The often equilateral triangle or isosceles triangle are used. This process is called as smoothing[2],[3]. The goal of smoothing is to obtain regular elements in mesh. However, in the solving domain such as finite element method, the more corrected results can be achieved.

There are many kinds of smoothing in the literature. These are; laplacian smoothing [4],[5],[6], optimization based smoothing, hybrid smoothing which is combining of laplacian and optimization based smoothing, angle based smoothing, variations of laplacian smoothing[7], winslow smoothing,..., etc. Although laplacian smoothing need computational cost less than other methods, invalid elements can be generated in mesh. On the other hand, while optimization ]based smoothing is more likely to avoid invalid elements, the computational costs is higher than laplacian smoothing. Hybrid methods eliminate generating of invalid elements of laplacian smoothing and is decreased computational cost of optimization based smoothing. But, mesh quality of hybrid methods is not as good as pure optimization based smoothing. Angle based smoothing provides good mesh quality, but it needs computational cost less than optimization based smoothing.

The common property of all methods is the working as global smoothing. That is, firstly mesh is created and then remeshing is done for smoothing. This situation causes more time and more computational cost.

In this study, while creating a mesh, the smoothed mesh is produced in 2D. So, remeshing process does not need. Fuzzy logic technique based a method is applied to 
smoothing process. It has known that, fuzzy logic technique has used firstly by [8]. First application areas are control applications. And then, different areas are applied to such as speech and pattern recognition, image processing,..., etc. Applications about computer graphics are curve modeling [9], curve smoothing and blending[10],...,etc.

The proposed method works more effective and more speed according to aforementioned methods. Due to the fact that fuzzy logic technique is used, the method does not need more computational cost. Also, quality of mesh according to discrete points on the boundary curve is attractivable. The only effected factor of mesh quality is the size of triangle which is dependent on distance of discrete points and angle of discrete points. Presented method is avoided invalid elements which is drawback of laplacian smoothing method. Due to the fact that smoothed mesh process is done and as possible as equilateral triangle or isosceles triangle is used, the method has some advantages. Method is detailed in section two and three.

The organization of paper is as follows; section two describes a presented method which is the based on fuzzy logic. In Section 3, fuzzy membership functions, rule base table and a simple application are given. At the finally conclusion is offered.

\section{Fuzzy Logic Based Smoothing}

In this section, fuzzy based smoothing method (FBSM) is described. The basis of presented method is to create equilateral triangle or isosceles triangle. The problem we have interested is to generate smoothed triangulation in 2D. The goal is to form equilateral triangle or isosceles triangle as possible as. It is known that, a good triangulation algorithm should be provided following criteria [11] ;

- The proposed must be general. That is, it must be independent on positions of triangles.

- After smoothing process has finished, unsmoothed element should not be remain of.

- It must be effective as computational cost and time requirements

- It must be easy-to-implement.

The presenting method provides above conditions. In the following section, the application method is gives detailed.

\subsection{Discretisation of Boundary Curve}

For this process, geometric support construction method is used. The set of polygonal segments of boundary curve is located in the any list. And then, the construction of the polygonal support is such that the distance any segment and portion of the curve it represent must be bounded. Hence, refinement of curve will be more important and correct in the any highly curved regions on the boundary curve.

Let be a distance between a point and the supporting edge, and let $\mathrm{b}$ be the length of the edge. If ration of $\mathrm{a} / \mathrm{b}$ is less than $\varepsilon$ we will determine, the segment we have selected is correct or wrong. If it is wrong, then segment is subdivided into sub- 
segments, each of them being analyzed recursively. As a result, we can make it geometric support. The ration $\mathrm{a} / \mathrm{b}$ is user-defined.
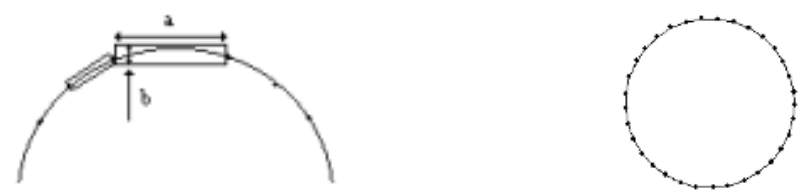

Fig. 1. a. Approximation using polygon segment b. Discretized boundary values of circle

\subsection{Locating of First Triangle}

The presenting method is similar to advancing front in way of starting process. So, first triangle is located between two nodes selected randomly from list.

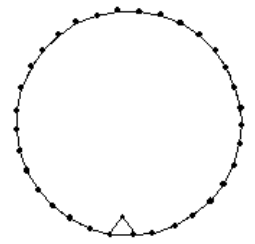

Fig. 2. Location of first triangle
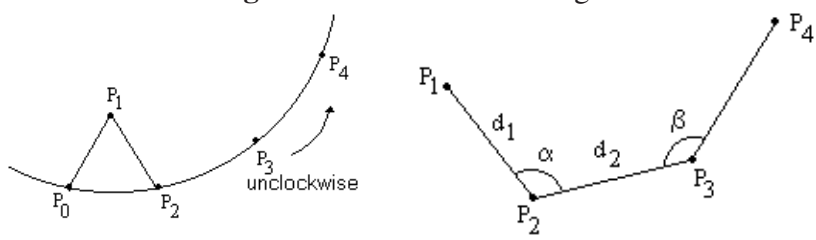

Fig. 3. a. Determining of list direction and node location for triangulation; b. Angles $\alpha$ and $\beta$, distances $d_{1}$ and $d_{2}$.

\subsection{Fuzzy Smoothed Triangulation Method in 2D}

Let points $\mathrm{P}_{0}, \mathrm{P}_{2}, \mathrm{P}_{3}, \mathrm{P}_{4}$ be discrete points and point $\mathrm{P}_{1}$ be a vertex of first triangle.

Firstly, triangle $\mathrm{P}_{0} \mathrm{P}_{1} \mathrm{P}_{2}$ is located as shown figure 3.a. Afterwards, the operation that will be done is that triangles are to produce by selecting node position in way of interior of closed region in unclockwise direction. For this process, the following steps are applied;

Step 1.

The first triangle is that nodes are $\mathrm{P}_{0} \mathrm{P}_{1} \mathrm{P}_{2}$. The purpose is to generate a node in such a way that, based edge of triangle is $\mathrm{P}_{2} \mathrm{P}_{3}$ and such that triangle to be created is equilateral or isosceles triangle. Let us draw a line dividing into two equal segment the angle $\alpha$. The goal is to find the best location on the line to be drawn form point $\mathrm{P}_{2}$. It must be found so point that, it will be proportional to distances $d_{1}$ and $d_{2}$. However, 
triangle to be created will be equilateral or isosceles triangle. Firstly, selection of $\mathrm{P}_{\mathrm{y}}$ seems to be reasonable at distance of d1 figure 4.b). The dark line depicts edges of a new triangle.
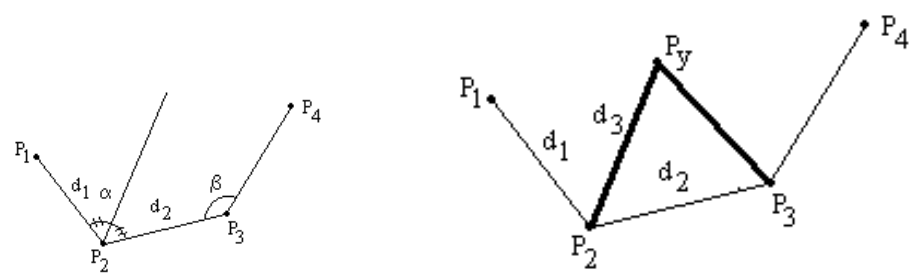

Fig. 4. a. Line drawing to divide into two equals segment the angle b. $\alpha$. The situation of $d_{3}$ equal $\mathrm{d}_{2}$
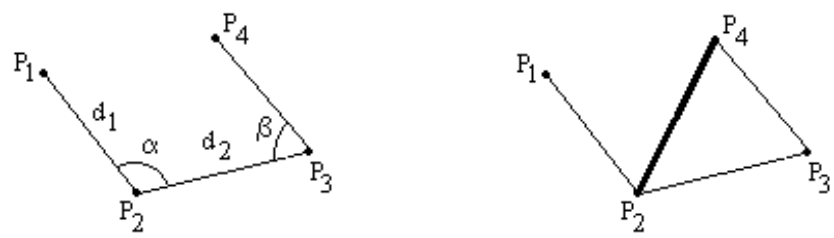

Fig. 5. a. The proposed method for $\beta<90$;

b. Triangle producing by connecting $\mathrm{P}_{2} \mathrm{P}_{4}$.

In this way, triangle to be produced is the isosceles at the worst case. But, distance d1 is not proportional $\mathrm{d}_{3}$. Therefore, unsmoothed triangles will occur when triangulation process is proceeded. To avoid this situation, distances $\mathrm{d} 3$ can be selected as half of summation of $\mathrm{d}_{1}$ and $\mathrm{d}_{2}$ (equation 1 ).

$$
d_{3}=\left(d_{1}+d_{2}\right) / 2
$$

Also, a different case may be position of $\mathrm{P}_{4}$ (figure 5.b). At this case, instead of finding a new $\mathrm{P}_{\mathrm{y}}$, directly triangulation is done.

In this case, triangle $\mathrm{P}_{2} \mathrm{P}_{3} \mathrm{P}_{4}$ is produced by connecting $\mathrm{P}_{2} \mathrm{P}_{4}$.

When the list has proceeded, same situation may appear while inner points are computed. Therefore, the position of $\mathrm{P}_{1} \mathrm{P}_{2} \mathrm{P}_{3}$ is important.

At this case, triangulation is done without computing a new point. The vertex of new triangle to be created is $\mathrm{P}_{1} \mathrm{P}_{2} \mathrm{P}_{3}$. (Fig. 6.b8).
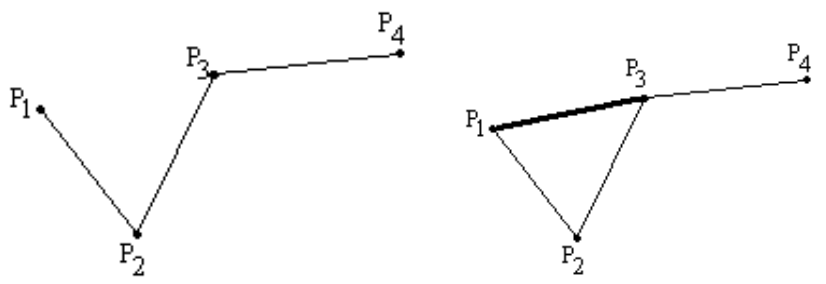

Fig. 6. a. Different $\mathrm{P}_{1} \mathrm{P}_{2} \mathrm{P}_{3}$ points; . b.A new triangle to be created

Noticed that, the basic parameters in the triangulation process are angle $\alpha, \beta$ and distances $d_{1}, d_{2}$. Therefore, a new method that will be developed by using angles $\alpha$ 
and $\beta$, distances $d_{1}$ and $d_{2}$ will provide fast and smoothing. For this process, fuzzy logic based a method is proposed in section3.

The following steps are shown the proposed method according to angles of $\alpha$ and $\beta$.

1. For $\alpha<90$; triangulation is performed without finding a new point(figure 7.a)

2. For $90=\alpha<180$; It is important the value of angle $\beta$.

a. For $\beta<=90$; two triangle is produced. The one of them is triangle $\mathrm{P}_{1} \mathrm{P}_{2}$ and other is $\mathrm{P}_{2} \mathrm{P}_{3} \mathrm{P}_{4}$ (figure 7.b)

b. For $\beta>90$; A new point is computed by means of fuzzy logic technique. If it is noticed to figure 11 , the position of $\mathrm{P}_{4}$ is not so important. The positions of $\mathrm{P}_{4}$ are shown as $\mathrm{P}_{4}, \mathrm{P}_{4},{ }^{\prime}, \mathrm{P}_{4},{ }^{\prime}$, .

Another situations of node positions may be find out similarly.
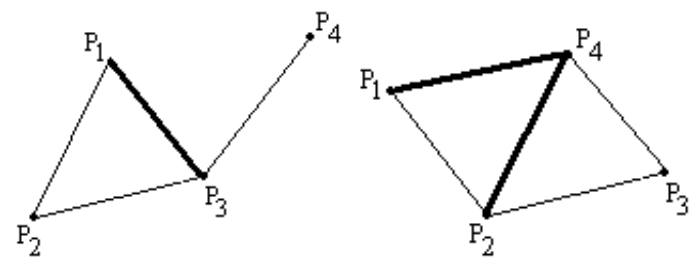

Fig. 7. a. Triangle for $\alpha<90$. b. Triangle for $\beta<=90$.

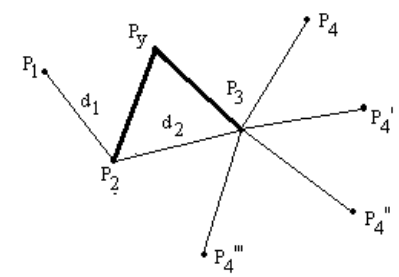

Fig. 8. A new triangle is triangle of $\mathrm{P}_{2} \mathrm{P}_{3} \mathrm{P}_{\mathrm{y}}$

\section{Membership Functions and Rule Base Table}

In this section, the membership functions and rule base table for fuzzy logic technique are given. Angle $\alpha$ and $\beta$ and distances $d_{1}$ and $d_{2}$ are used for optimal triangulation. Angles $\alpha$ and $\beta$ change in the interval $[90,360]$ according to boundary of object. Also, distances $\mathrm{d}_{1}$ and $\mathrm{d}_{2}$ can change random according to discretisation of boundary. Therefore, proportional of them is used (equation 2).

$$
\begin{array}{ll}
k=d_{1} / d_{2}, & \text { for } d_{1}>d_{2} \\
k=d_{2} / d_{1}, & \text { for } d_{1}>d_{1}
\end{array}
$$

If it is noticed to figure 9 , the constant $\mathrm{k}$ can seem as if it were changed in the large interval. Whereas, due to the fact that almost equal discretization is made, the changing of $\mathrm{k}$ will be in certain interval. 


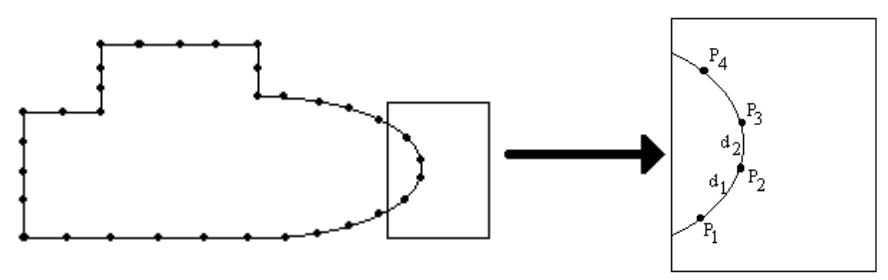

Fig. 9. The values of $d 1$ and $d 2$.
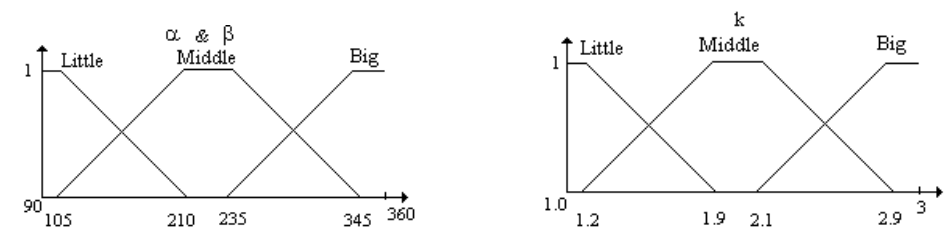

Fig. 10. Input membership functions

At this way, the tracing boundary and continuity of derivative would be provided. In this paper, the changing of $\mathrm{k}$ will be considered as [0.01,3]. At the other words, for $\mathrm{d}_{1}>\mathrm{d}_{2}, \mathrm{~d}_{1}$ will be three times of $\mathrm{d}_{2}$. It is known that, firstly, the determination of membership functions and rule base table is required for fuzzy logic technique. Angles $\alpha$ and $\beta$ and constant $\mathrm{k}$ are used as inputs of fuzzy logic. Figure 10 shows input membership functions.

Some outlines on developing the membership function for fuzzy sets are listed as follows;

1. Membership function as a rational function of polynominals,

2. Membership function as a model for human concepts,

3. Heuristically based membership functions,

4. Membership functions based on statistics or probability density functions [12].

In this paper, we concern with human concept based membership function.

Figure 11 shows output parameters of fuzzy logic. These are distance $d_{3}$ and angle of $\gamma$.

Figure 12 depicts output membership function.

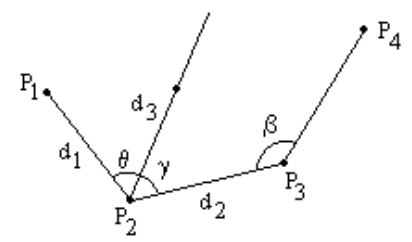

Fig. 11. Parameters using for output.
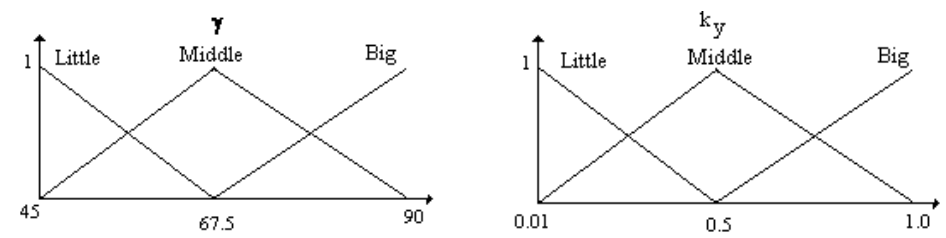

Fig. 12. Output membership function. 
Distance $\mathrm{d}_{3}$ is proportional $\mathrm{k}_{\mathrm{y}}$. Equation 3 depicts this relation.

$$
\begin{array}{ll}
d_{3}=k_{y} * d_{1}, & \text { for } d_{1}>d_{2} \\
d_{3}=k_{y} * d_{2}, & \text { for } d_{2}>d_{1}
\end{array}
$$

As defuzzyfication, middle of maximums are used in this study (Equation 4)

$u_{i}$ : $i^{\text {th }}$ value of membership function $u$.

$\mu\left(u_{i}\right)$ : The maximum value of membership function $u_{i}$.

$$
u^{*}=\frac{\sum_{i=1}^{l} \cdot \mu\left(u_{i}\right) \cdot u_{i}}{\sum_{i=1}^{l} \mu\left(u_{i}\right)}
$$

Table 1 shows rule base table for fuzzy logic.

Table 1.

\begin{tabular}{|c|c|c|c|c|c|c|c|c|c|}
\hline \multicolumn{3}{|c|}{ Inputs } & \multicolumn{2}{c|}{ Outputs } & \multicolumn{3}{c|}{ Inputs } & \multicolumn{2}{c|}{ Outputs } \\
\hline$\alpha$ & $\beta$ & $\mathbf{k}$ & $\gamma$ & $\mathbf{k}_{\mathbf{y}}$ & $\alpha$ & $\beta$ & $\mathbf{k}$ & $\gamma$ & $\mathbf{k}_{\mathbf{y}}$ \\
\hline 1 & 1 & 1 & 1 & 3 & 2 & 2 & 1 & 1 & 2 \\
\hline 1 & 1 & 2 & 1 & 1 & 2 & 2 & 2 & 1 & 1 \\
\hline 1 & 1 & 3 & 1 & 1 & 2 & 2 & 3 & 1 & 3 \\
\hline 1 & 2 & 1 & 1 & 3 & 3 & 3 & 1 & 1 & 2 \\
\hline 1 & 2 & 2 & 1 & 2 & 3 & 3 & 2 & 1 & 1 \\
\hline 1 & 2 & 3 & 1 & 1 & 3 & 3 & 3 & 1 & 2 \\
\hline 1 & 3 & 1 & 1 & 3 & 3 & 1 & 1 & 1 & 3 \\
\hline 1 & 3 & 2 & 1 & 1 & 3 & 1 & 2 & 1 & 2 \\
\hline 1 & 3 & 3 & 2 & 3 & 3 & 1 & 3 & 1 & 1 \\
\hline 2 & 1 & 1 & 2 & 2 & 3 & 2 & 1 & 1 & 3 \\
\hline 2 & 1 & 2 & 2 & 1 & 3 & 2 & 2 & 2 & 2 \\
\hline 2 & 1 & 3 & 1 & 3 & 3 & 2 & 3 & 1 & 1 \\
\hline
\end{tabular}

For $\alpha, \beta$ and $\mathrm{k}$; 1 : Little, 2: Middle and 3: Big. For $\gamma$ and $\mathrm{k}_{\mathrm{y}}$;1: Little, 2: Middle and 3: Big.

After computing angle of $\gamma$ and $\mathrm{k}_{\mathrm{y}}$, location of py is computed in simple analytical manner.

The following section describes how to apply of fuzzy logic and how to find of Py

\subsection{Application}

In the presented application, how fuzzy logic technique was applied to smoothing is described.

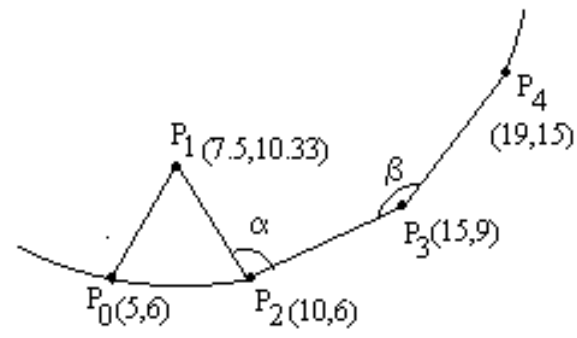

Fig. 13. An object which boundary was discreted 
The first step of proposed method is to locate first triangle. For this process, the first triangle is constructed as equilateral triangle. The next step is to compute angles $\alpha, \beta$ and distances $d_{1}, d_{2}$ These values are computed as $\alpha=98.42^{\circ}, \beta=171.9^{\circ}, d_{1}=4.89$ and $\mathrm{d}_{2}=5.83$.

$\gamma$ and $\mathrm{k}_{\mathrm{y}}$ values are computed by means of $\alpha=98.42^{\circ}$ ve $\beta=171.9^{\circ}$ ve $\mathrm{d}_{1}=4.89$ ve $\mathrm{d}_{2}=5.83$, which is inputs of fuzzy logic technique. These values are $\gamma=53.1$ and $\mathrm{k}_{\mathrm{y}}=0.736$ and $\mathrm{d}_{3}=0.735 * 5.83=4.29$. Figure 14 depicts position of $\mathrm{P}_{\mathrm{y}}$.

The method is iterated according to object boundary. Figure 15.a shows unsmoothed object and figure 15.b shows smoothed object.

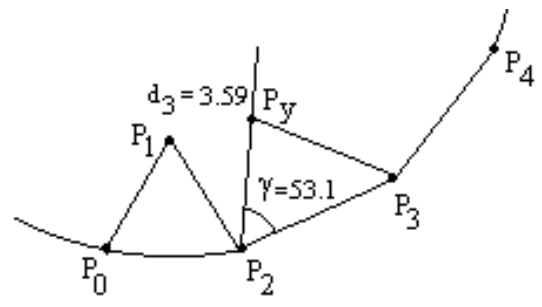

Fig. 14. The Computing value of $\mathrm{P}_{\mathrm{y}}$.
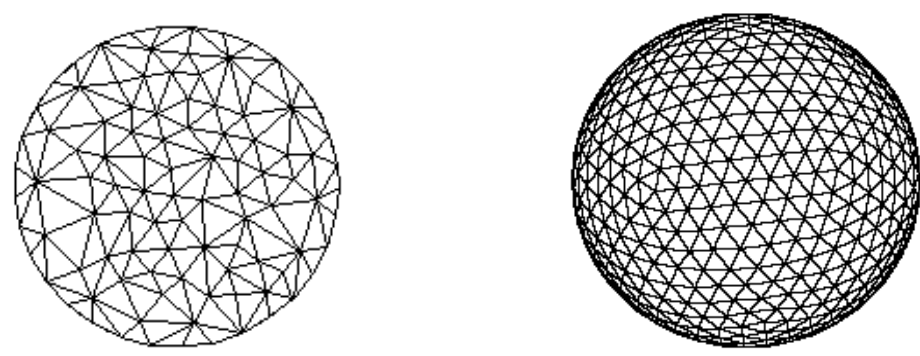

Fig. 15. a. Unsmoothed object b. Smoothed object.

\section{Conclusion}

In this study, a novel method based on fuzzy logic technique for smoothing in $2 \mathrm{D}$ is proposed. The use of fuzzy logic technique has provided only remeshing process for smoothing. Therefore, the proposed method is differ from other method in the literature. Due to the fact that smoothing process is achieved, remeshing process doesn't require. So, computational cost is decreased.

The proposed method has a little time requirements for fuzzy computation. This time is very little. In the proposed method, the changing of angle is not considered. As future work, the proposed method will be tried to be applied 3D object. 


\section{References}

1. Parhasarthy V., S. Kodiyalam, "A Constrained optimization approach to finite element mesh smoothing", Finite Elemnts in anlysis and Design, Vol: 9. Pp:309-320, 1991

2. Field D.A., "Laplacian Smoothing and Delaunay Triangulation", Communications in Applied Numerical Methods, Vol: 4, pp:709,712, 1988

3. Freitag L.A., "On Combining Laplacian and Optimization based Mesh Smoothing Techniques,"AMD Trends in Unstructured Mesh Generation, Vol:220, pp:37,43, 1997

4. Hansbo P, "Generalized Laplacian Smoothing of Unstructured Grids" Communication in Numerical Methods in Engineering, Vol: 11:pp:454-464, 1995

5. Zhao T., Shimada Kenji, "An Angle-based approach to two dimensional mesh smoothing", $9^{\text {th }}$ Int. Meshing Roundtable, pp:373-384, 2000

6.. Canann S., J.R. Tristano, M.L.Staten, "An Approach to Combined Laplacian and Optimization-Based Smoothing for Triangular, Quadrilateral and Quad-Dominant Meshes", $7^{\text {th }}$ Int. Meshing Roundtable, pp:479-494. 1998

7 Saga S. Makino H., "A Method for Modeling Freehand Curves-the Fuzzy Spline Interpolation", Systems and Computers in Japan, Vol: 26, No:19, pp:1610-1619, 1995.

8 Zadeh L. "Fuzzy sets “, Inf. Control, Vol:8, pp:338-353, 1965.

9 Gao Lun Shan, Kawarada Hideo, "Applications of fuzzy average to curve and surfaces fitting”, $2^{\text {nd }}$ Fuzzy Eng. Sym.IEEE Inter. Con. on Fuzzy systems, Vol. 2, p 971-978 , 1995.

10 Ahmet Çınar, Ahmet Arslan, "Blending of 2D Objects Using Fuzzy Logic", Lecture Notes in Computer Science, Vol: 2206, pp: 968-976, 2001

11 Owen S...'A Survey of Unstructured Mesh Generation Technology" 7h Int. Meshing Roundtable, pp:239-268, 1998

12 Parhasarthy V., S. Kodiyalam, "A Constrained optimization approach to finite element mesh smoothing", Finite Elemnts in anlysis and Design, Vol: 9. Pp:309-320, 1991 